This item was submitted to Loughborough's Research Repository by the author.

Items in Figshare are protected by copyright, with all rights reserved, unless otherwise indicated.

\title{
Hausdorff moments in an inverse problem for the heat equation: numerical
} experiment

PLEASE CITE THE PUBLISHED VERSION

LICENCE

CC BY-NC-ND 4.0

\section{REPOSITORY RECORD}

Kurylev, Y.V., N. Mandache, and K.S. Peat. 2019. "Hausdorff Moments in an Inverse Problem for the Heat Equation: Numerical Experiment”. figshare. https://hdl.handle.net/2134/449. 


\title{
Hausdorff moments in an inverse problem for the heat equation: Numerical experiment
}

\author{
Y.V. Kurylev, N. Mandache and K. S. Peat *
}

\section{Introduction}

Let $\Omega \subset \mathbf{R}^{n}$ be a bounded open set with piecewise smooth boundary. Consider the heat equation for the acoustic operator:

$$
\partial_{t} u=\rho^{-1} \Delta u \quad \text { in } \Omega \times \mathbf{R}_{+},
$$

with $C^{-1} \leq \rho \leq C, C>1$. When $n=3$ it describes the evolution of temperature $u$ in a medium where the thermal conductivity is constant and the heat capacity is $\rho$. It also applies to diffusion in porous rocks, where $\rho$ is the specific storage, if the hydraulic conductivity is constant.

We consider the problem of the determination of $\rho$ in $\Omega$ from boundary measurements. Consider the system:

$$
\left\{\begin{array}{l}
\Delta u(x, t)=\rho(x) \partial_{t} u(x, t) \\
u(x, 0)=0 \\
\left.u(t)\right|_{\partial \Omega}=\left.p\right|_{\partial \Omega} \text { for any } t \geq 0
\end{array}\right.
$$

where $p$ is a harmonic function. The solution, denoted $u_{p}$, is the temperature in the body $\Omega$ which was held at constant temperature $u=0$ before $t=0$ and to which the temperature $\left.p\right|_{\partial \Omega}$ is imposed at the boundary for $t \geq 0$. We will suppose that we can make a finite number of such experiments with different $\left.p\right|_{\partial \Omega}$ 's and each time measure the heat flux $\partial_{\nu} u_{p}$ on $\partial \Omega(\nu$ is the exterior normal to the boundary). As $t \rightarrow \infty, u_{p}(x, t)$ tends to the steady state $p(x)$. Take $p$ above to be a harmonic polynomial; let $q$ be another

${ }^{*}$ Department of Mathematical Sciences, Loughborough University, Loughborough, LE11 3TU, UK 
harmonic polynomial, and $T>0$ be the final time in our experiment. We define:

$$
\begin{aligned}
\Phi_{p, q}(T) & :=\int_{\Omega} u_{p}(x, T) q(x) \rho(x) d x=\int_{0}^{T} \int_{\Omega} q \rho \partial_{t} u_{p} d x d t \\
& =\int_{0}^{T} \int_{\Omega} q \Delta u_{p} d x d t=\int_{0}^{T} \int_{\partial \Omega}\left(q \partial_{\nu} u_{p}-u_{p} \partial_{\nu} q\right) d x d t \\
& =\int_{0}^{T} \int_{\partial \Omega}\left(q \partial_{\nu} u_{p}-p \partial_{\nu} q\right) d x d t=\int_{0}^{T} \int_{\partial \Omega} q \partial_{\nu}\left(u_{p}-p\right) d x d t
\end{aligned}
$$

so $\Phi_{p, q}(T)$ can be computed from boundary measurements.

Remark. It is seen from formula (3) that in order to find $\Phi_{p, q}(T)$ we need to know the total heat flow $\int_{0}^{T} \partial_{\nu} u_{p} d t$ through an elementary surface rather than the instantaneous heat flow $\partial_{\nu} u_{p}$.

Kawashita, Kurylev and Soga [4] have used the functional $\Phi_{p, q}(T)$ to obtain approximate values for the moments of $\rho$. First, they have shown that

$$
\left|\Phi_{p, q}(t)-M_{p, q}\right| \leq e^{-\lambda_{1} T}\|p\|_{L^{2}(\Omega, \rho)}\|q\|_{L^{2}(\Omega, \rho)}, \quad M_{p, q}:=\int_{\Omega} p q \rho d x
$$

where $\lambda_{1}$ is the first eigenvalue of $-\rho^{-1} \Delta$ with Dirichlet boundary conditions. This operator is self-adjoint in $L^{2}(\Omega, \rho)$ and $\lambda_{1} \geq \inf _{\Omega}\left(\rho^{-1}\right) \lambda_{1}^{0}$, where $\lambda_{1}^{0}$ is the first Dirichlet eigenvalue of $-\Delta$ in $\Omega$.

Then they used the fact that the products $p q$ of harmonic polynomials span the space of all polynomials, and gave a stability estimate and a reconstruction procedure for $\rho$. These are based on approximating the Gaussian bells $\mu^{-n} \exp \left(-\left(\frac{x-x_{0}}{\mu}\right)^{2}\right)$ by polynomials, and letting $\mu \rightarrow 0$.

The main task of the present work is to give a numerical application of these reconstruction procedures in the 2-dimensional case. However, instead of Gaussian bells, we will use an algorithm similar to that of Talenti [9] to solve a two-dimensional finite Hausdorff moments problem.

The moments method has been successfully applied to spectral inverse problems, in which eigenvalues and boundary traces of eigenfunctions are known. This was done first by Kurylev and Starkov [6], using directional moments. A numerical treatment was given by Kurylev and Peat [7], [8].

Let us describe briefly the plan of the paper and the numerical algorithm used. We will take $\Omega$ to be a square, $\Omega=(-1,1)^{2}$. In section 2 , using a semidiscrete finite element scheme we compute the solution $u$ for $2 m+1$ different boundary conditions $\left.p\right|_{\partial \Omega}$, with $p$ being harmonic polynomials, namely, the real and imaginary parts of $z^{0}, \ldots, z^{m}$, with $z=x+i y$. This allows us to 
compute $\Phi_{p, q}(T)$ when $\operatorname{deg} p \leq m$, which are approximately equal to $M_{p q}$ (4). Since, however, $\Phi_{p, q}(T) \neq M_{p q}$ we describe in section 3 an extrapolation procedure to construct $M_{p, q}^{*}$ which is a better appproximation to $M_{p, q}$ then $\Phi_{p, q}(T)$. This makes it possible to find approximate values, $M_{i j}^{*}$ of the moments $M_{i j}$ of $\rho$ :

$$
M_{i j}:=\int_{\Omega} \rho(x) x^{i} y^{j} d x d y
$$

for $i+j \leq 2 m$. In Section 4 , using $M_{i j}^{*}$ we approximately compute the first few Fourier-Legendre coefficients of $\rho$ and construct an approximation to $\rho$. Finally, in section 5 we discuss the general applicability of the method.

\section{Direct problem}

The direct problem was solved by the semi-discrete finite element method, i.e. with the time dimension approximated by finite differences. Domain $\Omega$ is subdivided into a finite number $N$ of subdomains $\Omega_{e}$ each with $m_{e}$ nodes and within each one we write

$$
u(x, t) \approx \sum_{i=1}^{m_{e}} N_{i}(x) a(t)=\mathbf{N} \mathbf{a}
$$

for known element shape functions $N_{i}(x)$. The weak Galerkin finite element formulation of (2) is then

$$
\sum_{e}\left(\int_{\Omega_{e}}\left[\nabla^{T} \mathbf{N} \nabla \mathbf{N a}+\mathbf{N}^{T} \rho \mathbf{N} \frac{\mathbf{a}}{d t}\right] d \Omega\right)=0 .
$$

The assembled set of equations for all elements can then be written in the matrix form

$$
C \frac{d \mathbf{a}}{d t}+K \mathbf{a}=\mathbf{0}
$$

For a time increment $\tau$, we write $f(n \tau)=f^{(n)}$ for the value of function at discrete time steps. The using Newmark's method

$$
C\left[\mathbf{a}^{(n+1)}-\mathbf{a}^{(n)}\right]+\tau K\left[\theta \mathbf{a}^{(n+1)}+(1-\theta) \mathbf{a}^{(n)}\right]=\mathbf{0}
$$

is used to advance the solution in time, and $\theta, 0<\theta<1$ is chosen to give anything between an explicit or fully implicit procedure.

In the example cases that follow in this paper, a mesh of 100 quadratic elements was used in the square domain $\Omega=(-1,1)^{2}$ with $\theta=0.5$ and $\tau=0.04$. 


\section{Extrapolation of data}

Due to the analyticity of the semigroup $t \rightarrow \exp \left(-t \rho^{-1} \Delta\right), \Phi_{p, q}(t)$ extends to an analytic function of $t$ for $\Re t>0$. Moreover, estimate (4) is valid for complex $t$ with $\Re t>0$ if we replace $t$ by $\Re t$ in the right hand side of this equation. This enables us to give a better approximation of $M_{p, q}$. In [4], the authors use Carleman quasi-analytic continuation of $\Phi_{p, q}(t), t \rightarrow \infty$. The corresponding integral representation that takes into account all the values of $\Phi_{p, q}(t)$ for $t \in[0, T]$ is

$$
\widetilde{M}(p, q)=\frac{e^{-\frac{1}{4}|\log \varepsilon|^{1 / 3}}}{2 \pi i} \int_{\pi / 2}^{3 \pi / 2} \Phi_{p, q}(t(w)) \varepsilon^{-\frac{1}{4}(w-1)^{2}} \frac{i w d \phi}{1-|\log \varepsilon|^{-1 / 3}-w},
$$

where $w=e^{i \phi}, t(w)=(1-w)^{-1} \sqrt{2\left(1+w^{2}\right)} T$ and a small parameter $\varepsilon=$ $O\left(\exp \left(-\lambda_{1} T\right)\right)$. However, due to the factor $\varepsilon^{-\frac{1}{4}(w-1)^{2}}$ this has proven to be unstable in numerical applications. We use a simpler approach that takes into account the exact form of $\Phi_{p, q}(t)$.

By substracting the stationary solution $u=p, w=u_{p}-p$ is the solution of the Cauchy problem (2) with $w(x, 0)=-p$ and $\left.w(t)\right|_{\partial \Omega}=0$. Denote by $\lambda_{j}, \phi_{j}$ the eigenvalues and eigenfunctions of $-\rho \Delta$ (with Dirichlet boundary conditions). Then $w(t)=-\sum_{j=1}^{\infty} \alpha_{j} \exp \left(-\lambda_{j} t\right) \phi_{j}$, with $\alpha_{j}=\int_{\Omega} p \phi_{j} \rho d x$. This shows that

$$
\Phi_{p, q}(t)=M_{p, q}-\sum_{1}^{\infty} a_{j} \exp \left(-\lambda_{j} t\right)
$$

with $a_{j}=\alpha_{j} \int_{\Omega} q \phi_{j} \rho d x$. Since $\lambda_{j} / j \rightarrow 4 \pi\left(\int_{\Omega} \rho d x\right)^{-1}$ by the Weyl asymptotics, the terms in (5) decay exponentially to zero when $t \rightarrow+\infty$ and the first term $a_{1} \exp \left(-\lambda_{1} t\right)$ is dominant at large $t$ when $a_{1} \neq 0$. We can therefore make a finite approximation

$$
\Phi_{p, q}(t) \approx M_{p, q}-\sum_{1}^{n} a_{j} \exp \left(-\lambda_{j} t\right) .
$$

Here we can suppose that all $\lambda_{j}$ are different. Indeed, if $\lambda_{j}=\lambda_{j+1}$ then we replace $a_{j}$ by $a_{j}+a_{j+1}$ and remove the $j+1$-th term. Also, we can suppose that $a_{j} \neq 0$ by re-enumerating $a_{j}, \lambda_{j}$.

Denote $f(t)=\sum_{1}^{n} a_{j} \exp \left(-\lambda_{j} t\right)$. Take $\tau>0$. We have

Theorem 1 If $a_{j} \neq 0$ and $\lambda_{j}<\lambda_{k}$ for $j<k$ then $f(0), f(\tau), \ldots, f((2 n-$ 1) $\tau$ ) completely determine $a_{j}$ and $\lambda_{j}$ for $j=1, \ldots, n$. 
Proof Denote $y_{j}:=\exp \left(-\tau \lambda_{j}\right), y_{j}>y_{k}$ for $j<k$. Then the numbers we know are $f(k \tau)=\sum a_{j} y_{j}^{k}$, for $k=0,1, \ldots, 2 n-1$. Take the Vandermonde matrix $V=\left(y_{k}^{j-1}\right)$. Here and below $j, k$ run from 1 to $n$. Since $y_{k}$ are all different, $V$ is non-singular. Consider now the linear system

$$
V^{t} \mathbf{b}=\mathbf{y}^{\mathbf{n}},
$$

where $\mathbf{b}=\left(b_{1}, \cdots, b_{n}\right)^{t}$ and $\mathbf{y}^{\mathbf{n}}=\left(y_{1}^{n}, \cdots, y_{n}^{n}\right)^{t}$. Since $V$ is non-singular, this system has a unique solution $\mathbf{b}$, with the property that all $y_{k}, k=1, \ldots, n$, are different roots of the polynomial equation

$$
y^{n}=\sum_{1}^{n} b_{j} y^{j-1} .
$$

This means that knowing $\mathbf{b}$ we can determine $y_{k}, k=1, \cdots, n$ by solving (8). Now, if we multiply from the left the both sides of (7) by the non-singular matrix $V \operatorname{diag}\left(a_{k}\right)$, we obtain the $n \times n$ system:

$$
f((n+k-1) \tau)=\sum_{j=1}^{n} f((j+k-2) \tau) b_{j}, \quad k=1, \cdots, n .
$$

This shows that we can obtain the vector $\mathbf{b}=\left(b_{1}, \cdots, b_{n}\right)$ from our data, and this gives $y_{k}, k=1, \cdots, n$. The vector $\mathbf{a}=\left(a_{1}, \cdots, a_{n}\right)^{t}$ can then be obtained from the equation $V \mathbf{a}=\mathbf{f}=\left(f(0), \cdots, f((n-1) \tau)^{t}\right.$.

In practice, this inversion is ill-posed, first because a Vandermonde matrix has a big condition number, and second, because solving the algebraic equation (8) to find $y_{k}$ is also ill-posed. If we merely want to extrapolate $f$, i.e., find the value of $f(k \tau)$ for a $k>2 n-1$, then we can proceed as follows. We find $\mathbf{b}$ using formula (9) above. We then use the fact that formula (9) remains valid for $k>n$. This gives a procedure to recurrently find $f(k \tau)$ ) for $k \geq 2 n$. Thus we need to solve only the linear system (9) and avoid solving the algebraic equation (8) and a second linear system $V \mathbf{a}=\mathbf{f}$. The numbers $b_{j}$ are the coefficients of the linear relation (9) which may be considered as a recurrence relation for $k \in \mathbb{N}$ that is satisfied by the sequence $f(j \tau), j \in \mathbb{N}$. In this respect, the above theorem is related to Berlekamp's algorithm, well-known in code theory.

In our case, (6) contains an unknown constant $M_{p, q}$. The theorem applies if we know the value of the sum in $(2 n+1)$ points. We first eliminate the constant by taking $\widetilde{f}(k \tau)=f(k \tau)-f((k+1) \tau)$ so that $a_{j}$ become $\widetilde{a}_{k}=a_{k}\left(1-y_{k}\right)$ where $y_{k}<1$ due to $\lambda_{k}>0$. We find out $\widetilde{a}_{k}, y_{k}$ and then determine the constant $M_{p, q}$ in (6). 
For a square (with $\rho \equiv 1$ ) $\lambda_{2} / \lambda_{1}=2.5$; in the variable $\rho$ cases that we study, $\lambda_{2} \in\left(2.3 \lambda_{1}, 2.5 \lambda_{1}\right)$, so the first term in the sum in (6) is dominant. This term can be determined approximately from the values of $\Phi_{p, q}(t)$ at three points equally spaced. Indeed, it is seen easily that using an approximation

$$
\Phi_{p, q}(t) \approx M_{p, q}-a_{1} \exp \left(-\lambda_{1} t\right)
$$

we obtain

$$
\exp \left(-\lambda_{1} \tau\right) \approx \frac{\Phi_{p, q}((j+1) \tau)-\Phi_{p, q}(j \tau)}{\Phi_{p, q}(j \tau)-\Phi_{p, q}((j-1) \tau)}
$$

However, for some $(p, q)$ the corresponding $a_{1}$ may be very small or even 0 so that the right-hand side in the above equation is not well-defined. Nevertheless, we can use this formula for $\Phi_{1,1}(t)$ since the corresponding $a_{1}>0$ is not small (actually $a_{1}=O\left(\rho_{\min } / \lambda_{1}\right)$ ). Having found $\exp \left(-\lambda_{1} \tau\right)$ we then evaluate an approximation $M_{p, q}^{*}$ to $M_{p, q}$ by

$$
M_{p, q}^{*}=\frac{\Phi_{p, q}((j+1) \tau)-\exp \left(-\lambda_{1} \tau\right) \Phi_{p, q}(j \tau)}{1-\exp \left(-\lambda_{1} \tau\right)} .
$$

In Table 1 the moments of $\rho_{1}=2+\cos \left(\frac{3}{2}(x+1)(y+1)\right)$ are plotted. The first column represents the exact values of the corresponding moments $M_{p, q}$ for $p, q=1, x, y, x y$ and $x^{2}-y^{2}$. The second column gives the values of the error $M_{p, q}-\Phi_{p, q}(T)$ at $T=0.8$. The third column shows the relative errors (in per cent). The forth column gives the values of the error after extrapolation and the last column shows the relative errors after extrapolation.

It is seen that, in general, extrapolation decreases relatively large errors but may increase relatively small ones. This corresponds to the fact that large errors occur when the exposure of the corresponding moment to the first eigenvalue is high, which is taken care of by the extrapolation procedure. When the error at the final time is relatively small, this indicates the larger effect of the second and further eigenvalues. Clearly, in this case extrapolation using the first eigenvalue would not decrease the overall error. More precisely, it follows from (5), (10) that the error after extrapolation is

$$
\frac{\sum_{2}^{\infty} a_{k} e^{-\lambda_{k} j \tau}\left(e^{-\lambda_{k} \tau}-e^{-\lambda_{1} \tau}\right)}{1-e^{-\lambda_{1} \tau}} .
$$

In comparison with (5) the actual error could be multiplied by a factor $\left(1-e^{-\lambda_{1} \tau}\right)^{-1}$ which, in our examples, can be up to the magnitude of 5 . 


\begin{tabular}{|l|l|l|l|l|l|l|}
\hline$p$ & $q$ & $\begin{array}{l}\text { exact } \\
\text { moment }\end{array}$ & $\begin{array}{l}\text { error before } \\
\text { extrapolation }\end{array}$ & $\begin{array}{l}\text { relative } \\
\text { error }\end{array}$ & $\begin{array}{l}\text { error after } \\
\text { extrapolation }\end{array}$ & $\begin{array}{l}\text { relative } \\
\text { error }\end{array}$ \\
\hline 1 & 1 & 8.9498 & 0.9114 & 10.2 & 0.0203 & 0.2 \\
\hline 1 & $x$ & -0.9409 & -0.1400 & 14.8 & -0.0109 & 1.2 \\
\hline 1 & $y$ & -0.9409 & -0.1400 & 14.8 & -0.0109 & 1.2 \\
\hline 1 & $x y$ & -0.1333 & 0.0003 & -0.2 & 0.0036 & -2.7 \\
\hline 1 & $x^{2}-y^{2}$ & 0.0000 & 0.0000 & 0 & 0.0000 & 0 \\
\hline$x$ & 1 & -0.9409 & -0.0724 & 7.7 & 0.0044 & -0.5 \\
\hline$x$ & $x$ & 3.1513 & 0.0276 & 0.9 & -0.0404 & -1.3 \\
\hline$x$ & $y$ & -0.1333 & 0.0075 & -5.7 & 0.0063 & -4.7 \\
\hline$x$ & $x y$ & -0.1701 & -0.0029 & 1.7 & 0.0063 & -3.7 \\
\hline$x$ & $x^{2}-y^{2}$ & -0.3491 & -0.0042 & 1.2 & 0.0092 & -2.6 \\
\hline$y$ & 1 & -0.9409 & -0.0724 & 7.7 & 0.0044 & -0.5 \\
\hline$y$ & $x$ & -0.1333 & 0.0075 & -5.7 & 0.0063 & -4.7 \\
\hline$y$ & $y$ & 3.1513 & 0.0276 & 0.9 & -0.04004 & -1.3 \\
\hline$y$ & $x y$ & -0.1701 & -0.0029 & 1.7 & 0.0063 & -3.7 \\
\hline$y$ & $x^{2}-y^{2}$ & 0.3491 & 0.0042 & 1.2 & -0.0092 & -2.6 \\
\hline$x y$ & 1 & -0.1333 & 0.0047 & -3.5 & 0.0001 & -0.1 \\
\hline$x y$ & $x$ & -0.1701 & -0.0025 & 1.5 & 0.0043 & -2.5 \\
\hline$x y$ & $y$ & -0.1701 & -0.0025 & 1.5 & 0.0043 & -2.5 \\
\hline$x y$ & $x y$ & 1.1478 & 0.0010 & 0.1 & -0.0040 & -0.3 \\
\hline$x y$ & $x^{2}-y^{2}$ & 0.0000 & 0.0000 & 0 & 0.0000 & 0 \\
\hline$x^{2}-y^{2}$ & 1 & 0.0000 & 0.0000 & 0 & 0.0000 & 0 \\
\hline$x^{2}-y^{2}$ & $x$ & -0.3491 & -0.0026 & 0.7 & 0.0056 & -1.6 \\
\hline$x^{2}-y^{2}$ & $y$ & 0.3491 & 0.0026 & 0.7 & -0.0056 & -1.6 \\
\hline$x^{2}-y^{2}$ & $x y$ & 0.0000 & 0.0000 & 0 & 0.0000 & 0 \\
\hline$x^{2}-y^{2}$ & $x^{2}-y^{2}$ & 1.5558 & 0.0011 & 0.1 & -0.0039 & -0.2 \\
\hline & & & & & & \\
\hline & & & & & \\
\hline & & & & & \\
\hline
\end{tabular}

Table 1: $\rho_{1}=2+\cos \left(\frac{3}{2}(x+1)(y+1)\right), T=0.8$.

However, when time increases, i.e. $j \rightarrow \infty$, the relative error due to higher eigenvalues exponentially decays with respect to one due to $\lambda_{1}$. For this reason and because the approximation to $\exp \left(-\lambda_{1} \tau\right)$ by formula (11) is better when $j$ is large, extrapolation works even better for large times. Indeed, as seen from Table 1, extrapolation has decreased the maximal relative error from 14.8 per cent to 4.7 per cent, i.e. by a factor of 3.14. Table 2 presents results (for $p, q=1, x, y$ ) when $T=1.28$ (with the same time step $\tau$ ). As it is seen the error has gone down from 4.8 percent to 0.6, i.e. 8 times. 


\begin{tabular}{|l|l|l|l|l|l|l|}
\hline$p$ & $q$ & $\begin{array}{l}\text { exact } \\
\text { moment }\end{array}$ & $\begin{array}{l}\text { error before } \\
\text { extrapolation }\end{array}$ & $\begin{array}{l}\text { relative } \\
\text { error }\end{array}$ & $\begin{array}{l}\text { error after } \\
\text { extrapolation }\end{array}$ & $\begin{array}{l}\text { relative } \\
\text { error }\end{array}$ \\
\hline 1 & 1 & 8.9498 & 0.2884 & 3.2 & -0.0000 & 0.0 \\
\hline 1 & $x$ & -0.9409 & -0.0449 & 4.8 & -0.0001 & 0.1 \\
\hline 1 & $y$ & -0.9409 & -0.0449 & 4.8 & -0.0001 & 0.1 \\
\hline$x$ & 1 & -0.9409 & -0.0223 & 2.4 & 0.0005 & -0.1 \\
\hline$x$ & $x$ & 3.1513 & 0.0045 & 0.1 & -0.0038 & -0.1 \\
\hline$x$ & $y$ & -0.1333 & 0.0032 & -2.4 & 0.0008 & -0.6 \\
\hline$y$ & 1 & -0.9409 & -0.0223 & 2.4 & 0.0005 & -0.1 \\
\hline$y$ & $x$ & -0.1333 & 0.0032 & -2.4 & 0.0008 & -0.6 \\
\hline$y$ & $y$ & 3.1513 & 0.0045 & 0.1 & -0.0038 & -0.1 \\
\hline
\end{tabular}

Table 2: $\rho_{1}=2+\cos \left(\frac{3}{2}(x+1)(y+1)\right), T=1.28$.

The same impact of extrapolation is seen from the next two tables which correspond respectively to the discontinuous case $\rho_{2}=1$ except for the square $0.25 \leq x, y \leq 0.75$ and the circle of the radius 0.25 with its center at $(-0.5,-0.5)$ where $\rho_{2}=2$, and continuous case $\rho_{3}=4\left(1+\sin \left(\frac{x+1}{2}\right)\right) /(5+$ $y)$.

\begin{tabular}{|l|l|l|l|l|l|l|}
\hline$p$ & $q$ & $\begin{array}{l}\text { exact } \\
\text { moment }\end{array}$ & $\begin{array}{l}\text { error before } \\
\text { extrapolation }\end{array}$ & $\begin{array}{l}\text { relative } \\
\text { error }\end{array}$ & $\begin{array}{l}\text { error after } \\
\text { extrapolation }\end{array}$ & $\begin{array}{l}\text { relative } \\
\text { error }\end{array}$ \\
\hline 1 & 1 & 4.4464 & 0.0800 & 1.8 & -0.0035 & -0.1 \\
\hline 1 & $x$ & 0.0158 & 0.0004 & 2.2 & -0.0001 & -0.3 \\
\hline 1 & $y$ & 0.0158 & 0.0004 & 2.2 & -0.0001 & -0.3 \\
\hline$x$ & 1 & 0.0158 & 0.0001 & 0.3 & -0.0001 & -0.3 \\
\hline$x$ & $x$ & 1.4529 & -0.0002 & 0 & -0.0011 & -0.1 \\
\hline$x$ & $y$ & 0.1116 & 0.0000 & 0 & -0.0002 & -0.2 \\
\hline$y$ & 1 & 0.0158 & 0.0001 & 0.3 & -0.0001 & -0.3 \\
\hline$y$ & $x$ & 0.1116 & 0.0000 & 0.0 & -0.0002 & -0.2 \\
\hline$y$ & $y$ & 1.4529 & -0.0002 & 0.0 & -0.0011 & -0.1 \\
\hline
\end{tabular}

Remark. The errors in Tables 3 and 4 are relatively small in comparison with Table 1 because $\lambda_{1}$ for $\rho_{2}, \rho_{3} \approx \pi^{2} / 2$ while for $\rho_{1} \approx \pi^{2}$.

Table 2: Discontinuous $\rho_{2}, T=0.8$. 


\begin{tabular}{|l|l|l|l|l|l|l|}
\hline$p$ & $q$ & $\begin{array}{l}\text { exact } \\
\text { moment }\end{array}$ & $\begin{array}{l}\text { error before } \\
\text { extrapolation }\end{array}$ & $\begin{array}{l}\text { relative } \\
\text { error }\end{array}$ & $\begin{array}{l}\text { error after } \\
\text { extrapolation }\end{array}$ & $\begin{array}{l}\text { relative } \\
\text { error }\end{array}$ \\
\hline 1 & 1 & 4.7349 & 0.1175 & 2.5 & -0.0033 & -0.1 \\
\hline 1 & $x$ & 0.4627 & 0.0139 & 3.0 & -0.0001 & 0.0 \\
\hline 1 & $y$ & -0.3191 & -0.0098 & 3.1 & 0.0000 & 0.0 \\
\hline$x$ & 1 & 0.4627 & 0.0071 & 1.5 & -0.0002 & 0.0 \\
\hline$x$ & $x$ & 1.5613 & 0.0008 & 0.1 & -0.0015 & -0.1 \\
\hline$x$ & $y$ & -0.0312 & -0.0006 & 1.9 & 0.0001 & -0.2 \\
\hline$y$ & 1 & -0.3191 & -0.0049 & 1.5 & 0.0001 & 0.0 \\
\hline$y$ & $x$ & -0.0312 & -0.0006 & 1.9 & 0.0001 & -0.2 \\
\hline$y$ & $y$ & 1.5955 & 0.0005 & 0.0 & -0.0016 & -0.1 \\
\hline
\end{tabular}

Table 3: $\rho_{3}=4\left(1+\sin \left(\frac{x+1}{2}\right)\right) /(5+y), T=0.8$.

\section{Finite Hausdorff moments' problem}

Consider the moments $M_{j k}=\int x^{j} y^{k} \rho d x d y$. If $\rho d x d y$ is a finite measure in $(-1,1)^{2}$ then $M_{j k}$ uniquely determine $\rho$, because the set of polynomials is dense in the set of continuous functions, which is the pre-dual of the set of finite measures.

The finite Hausdorff moments problem in dimension 1 consists in retrieving $\rho$ (approximately) from the moments $M_{0}, M_{1}, \ldots, M_{n}$. Talenti [9] considered this problem on the interval $(0,1)$. We use precisely the same approach, altered only to consider the interval $(-1,1)$. Thus in one dimension, given a moment

$$
\mu_{k}=\int_{-1}^{1} x^{k} \rho(x) d x
$$

it follows that

$$
\rho(x)=\sum_{i} \lambda_{i} L_{i}(x),
$$

where $L_{i}(x)$ are Legendre polynomials and the constants $\lambda_{i}$ are the FourierLegendre coefficients, which are related to the moments through

$$
\lambda_{i}=\sum_{j=0}^{i} C_{i j} \mu_{j}
$$

and $C_{i j}$ are the coefficints of the Legendre polynomials.

Results for the reconstruction of $\rho_{1}$ and $\rho_{2}$ using the first 16 moments are shown in Figures 1, 2 and Figure 3 respectively. For the smooth case, 

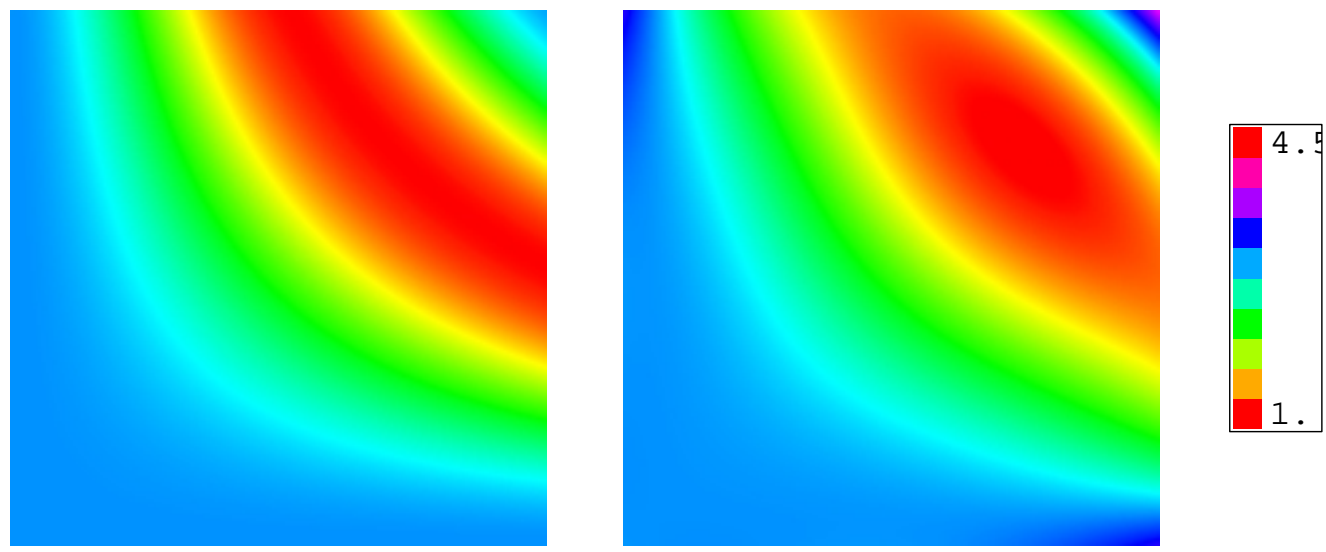

Figure 1: Reconstruction of a smooth $\rho_{1}$ using 16 moments. Left: Actual distribution. Right: Reconstruction using exact moments
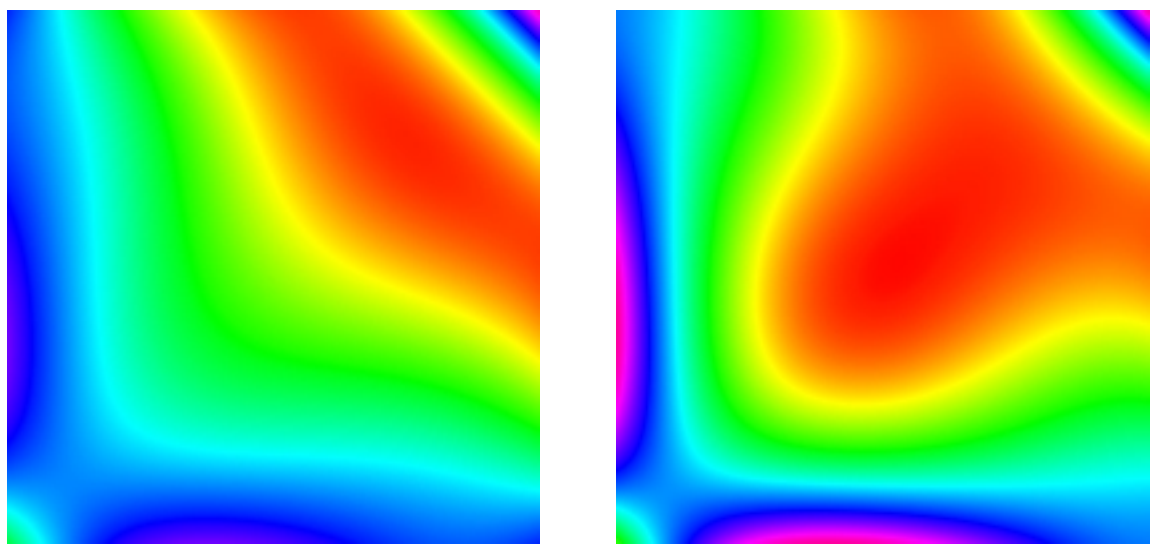

Figure 2: Reconstruction of a smooth $\rho_{1}$ using 16 moments. Left: Reconstruction using extrapolation from $T=0.8$. Right: Reconstruction from truncation at $T=0.8$. 

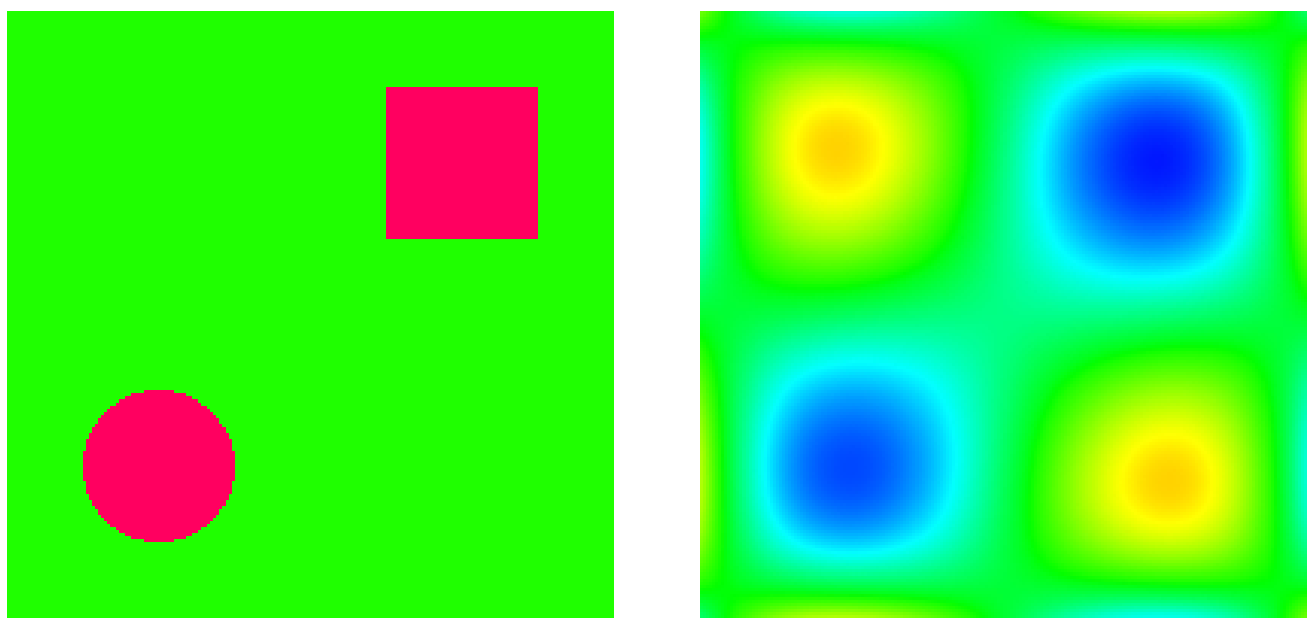

Figure 3: Reconstruction of a non-smooth $\rho_{2}$. Left: Actual distribution. Right: Reconstruction using extrapolation from $T=0.8$.

$\rho_{1}$, three different reconstructions are given, namely using exact moments, using moments resulting from extrapolation of data beyond $T=0.8$ as in section 3 , and using moments when resulting from truncating the analysis at $T=0.8$.

It is seen that even 16 exact moments can provide a fairly accurate reconstruction of a smooth distribution $\rho$. Furthermore, the extra accuracy in the moments from the use of extrapolation as compared to truncation at finite time yields a significant increase in the accuracy of reconstruction, as seen by comparison of Figures 2(left) and 2(right). Indeed, the reconstruction using moments from extrapolation is little worse then that using exact moments, see Figures 1(right) and 2(left).

In contrast, the reconstruction of the discontinuous $\rho_{2}$ is much worse, as seen in Fugure 3. Figure 3(right) is actually the reconstruction from moments evaluated by extrapolation, but there is no discernible difference between this reconstruction and those using exact moments or moments from a truncated analysis. In essence, the lowest moments are evaluated well enough by any method and the error is basically due to the neglect of higher order moments. 


\section{Conclusions}

As seen from the results of this paper the moments' method for the heat/diffusion equation (1) is quite efficient and, in the case of a smooth although highly variable $\rho$, provides a relatively good approximation of this unknown coefficient. It is important to mention that to obtain such an approximation the moments' method requires relatively few boundary measurements (in our case we have used just 7 boundary sources). An additional advantage in the case of a parabolic equation is in the exponential convergence of $\int_{\Omega} u_{p}(t) q \rho$ to $M_{p q}$ which has made it possible to use an exponential extrapolation to get a better approximation to the moments.

The precison of the method declines for non-smooth $\rho$ 's as is seen from the comparison of Figure 2 with Figure 3. This effect is natural because using the polynomial moments corresponds to the expansion of $\rho$ in the smooth basis of Legendre polynomials. Therefore, to get the same error as for a smooth $\rho$ we would need to have at our disposal significantly more moments, i.e. to have more boundary measurements. There is a partial remedy of this effect which may be used when we know a priori that the media consists of several components with distinctively different parameters like in the case of $\rho_{2}$. This idea is borrowed from the linear sampling method aimed at the reconstruction of a scatterer from scattering data and is in the choice of some level set of the reconstructed approximation to be the boundary between the components (e.g. [5], [1]). In Figure 4 we have used this idea to obtain the domains occupied by inclusions with $\rho=2$ for the inverse problem with unknown $\rho_{2}$. We note that as seen from relation (5) and positivity of $\phi_{1}$, the moments' method for the heat equations tends to descrease $M_{0}=\int_{\Omega} \rho$. Therefore we have chosen the level $\rho=1.35$ rather then $\rho=1.5$ for the boundary between components.

In order to improve the result we can increase the number of the moments used. However, as is shown in [7], [8], the improvement is painfully slow due to the logarithmic nature of convergence of the method. On the other hand, existing approximate reconstruction techniques like iteration methods (e.g. [2]) provide rather effective algorithms to solve inverse problems numericallly as soon as the initial approximation ("initial guess") is reasonable. In this connection, we believe that it might be useful to develop a combined method for solving inverse problems. The initial step would consist of an approximate solution of a fully-nonlinear inverse problem for (1) by the moments' method. The result would then been used as an initial guess for an iteration-type procedure to improve the quality of the reconstruction. Based on our numerical results we believe this may be efficient 

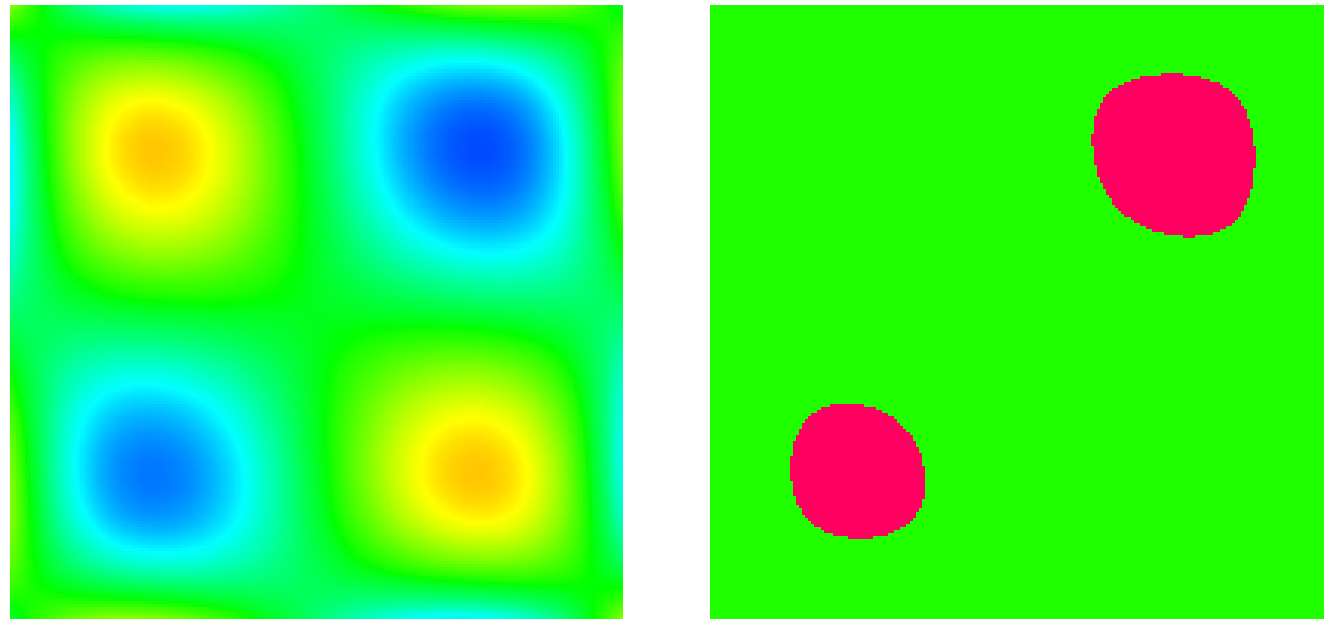

Figure 4: Reconstruction of a non-smooth $\rho_{2}$. Left: Reconstructed approximation of $\rho_{2}$ by Lagrange polynomials. Right: Piecewise-constant approximation.

for the reconstruction of both smooth and piecewise-smooth parameters $\rho$.

ACKNOWLEDGEMENTS The research of YVK and KSP were partly and of NM fully supported through EPSRC grant GR/M11463.

\section{References}

[1] Colton D., Kirsch A. and Monk P. The linear sampling method in inverse scattering theory. Surveys on solution methods for inverse problems, 107-118, Springer, Vienna, 2000.

[2] Engl H. W., Hanke M. and Neubauer A. Regularization of Inverse Problems. Mathematics and Applications , 375. Kluwer, Dordrecht, 1996. $321 \mathrm{pp}$.

[3] Inglese G., A note about the discretization of finite moment problems, Inverse Problems 10(1994), 401-414.

[4] Kawashita M., Kurylev Y. and Soga H., Directional moments for an inverse problem for heat equation, SIAM J. Math. Analysis, 32(2000), $522-537$. 
[5] Kirsch, A. Characterization of the shape of a scattering obstacle using the spectral data of the far field operator. Inverse Problems 14 (1998), 1489-1512.

[6] Kurylev Y. and Starkov A., Directional moments in the acoustic inverse problem, Inverse Problems in Wave Propagation, G. Chavent \& al (ed.), IMA Vol. in Mathematics and its Applications, Springer, 1997, 295-324.

[7] Kurylev Y. and Peat K. S., Hausdorff moments in 2-D inverse acoustic problems, Inverse Problems 13(1997), 1363-1377.

[8] Peat K.S., Kurylev Y. Hausdorff moments method of acoustic imaging. Acoustic Imaging 24 (2000). Kluwer, 79-86.

[9] Talenti G., Recovering a function from a finite number of moments, Inverse Problems 3(1987), 501-517. 